\title{
Uma revisão sistemática da literatura sobre a classificação de estilos de aprendizagem utilizando técnicas inteligentes
}

\section{A systematic literature review about classification of learning styles using intelligent techniques}

\author{
Denilson Gabriel Freitas de Carvalho ${ }^{1 *}$, Angelo Gabriel Lopes da Silva ${ }^{1}$, Gabriel Caldas Barros \\ e Sá ${ }^{1}$, Náthalee Cavalcanti de Almeida Lima ${ }^{1}$, Samara Martins Nascimentos ${ }^{1}$
}

\begin{abstract}
RESUMO
Os Estilos de Aprendizagem são um tema de estudo relevante, pois o conhecimento acerca disso proporciona aos professores e alunos um caminho para a melhoria do ensino e aprendizagem. Esta Revisão Sistemática da Literatura foi realizada com o objetivo de averiguar quais as propostas e abordagens automáticas são utilizadas para detecção/predição de Estilos de Aprendizagem. Foram selecionados 59 trabalhos das principais bases de dados, dos quais foram extraídos e sintetizadas informações acerca das técnicas inteligentes abordadas, seu desempenho e as métricas aplicadas para medi-lo, os atributos que serviram de entrada para o algoritmo, as plataformas usadas para implementálos e os modelos de Estilos de Aprendizagem mais empregados. A partir dos resultados observados, trabalhos futuros buscarão propor uma nova abordagem para predizer Estilos de Aprendizagem.
\end{abstract}

Palavras-chave: Estilos de Aprendizagem; Inteligência Computacional; Protocolo de Kitchenham; Revisão Sistemática da Literatura; Técnicas Inteligentes.

\begin{abstract}
Learning Styles are a relevant study topic because the knowledge about this provides a way for teachers and students to improve teaching and learning. This Systematic Literature Review was carried out with the objective of ascertaining which proposals and automatic approaches are used for the detection/prediction of Learning Styles. 59 works were selected of the main databases and, from these articles, information about the smart techniques addressed, their performance and the metrics applied to measure it were extracted and synthesized, the attributes that served input for the algorithm, the platforms used to implement them and the most used Learning Styles models. Based on the observed results, future work will seek to propose a new approach to predict Learning Styles.
\end{abstract}

Keywords: Computational Intelligence; Learning Styles; Kitchenham Protocol; Smart Techniques; Systematic Literature Review.

${ }^{1}$ Universidade Federal Rural do Semi-Árido (UFERSA).

"E-mail: nathalee.almeida@ufersa.edu.br 


\section{INTRODUÇÃO}

Os Estilos de Aprendizagem (EAs) são padrões que indicam como o aluno assimila conhecimentos, bem como relatam o indicativo sobre a maneira mais proveitosa para obtenção, processamento e armazenamento de informações. Além disso, os EAs podem ser considerados métodos mais adequados para explicitar as maneiras de como um aluno se concentra, memoriza novas informações e recorda informações antigas (DUTSINMA; TEMDEE, 2020)

A proposta tradicional para classificar os EAs envolve a resolução de questionário pelos alunos. No entanto, essa abordagem apresenta alguns problemas, dado que o processo de resolução do questionário torna-se enfadonho devido a grande quantidade de questões (EL AISSAOUI et al., 2019). Para solucionar tais problemas desenvolveram-se abordagens automáticas, sendo algumas delas elaboradas por meio de Inteligência Computacional (IC), que objetivam detectar os EAs dos alunos mediante seu comportamento e sua interação com um determinado sistema. Algumas das vantagens em comparação ao método convencional é que não há perda de tempo com processo de resolução de questionários (EL AISSAOUI et al., 2019).

A partir da importância da concepção relacionada aos EAs dos alunos e da viabilidade das abordagens automáticas que utilizam técnicas de inteligência computacional para realizar a classificação dos EAs, este trabalho tem como objetivo averiguar o estado da arte a respeito da classificação dos EAs por meio de Inteligência Artificial (IA). Para alcançar este objetivo, realizou-se uma Revisão Sistemática da Literatura (RSL), com base no protocolo proposto por Kitchenham e Charters (2007).

Para melhor compreensão, a organização deste trabalho se constitui da seguinte maneira: A Seção 2 descreve a metodologia seguida para o desenvolvimento da revisão sistemática; a Seção 3 discute os resultados obtidos com a RSL; e a Seção 4 apresenta as considerações finais deste trabalho.

\section{METODOLOGIA}

A Revisão Sistemática da Literatura é um meio de identificar, avaliar e interpretar todas as pesquisas disponíveis relevantes para uma questão de pesquisa específica, ou área de tópico, ou fenômeno de interesse (KITCHENHAM; CHARTERS, 
2007). As três principais fases da RSL são: Planejamento, Condução e Relato. O fluxograma mostrado na Figura 1 elenca as três etapas seguidas nesta RSL.

Figura 1 - Etapas seguidas na Revisão Sistemática da Literatura
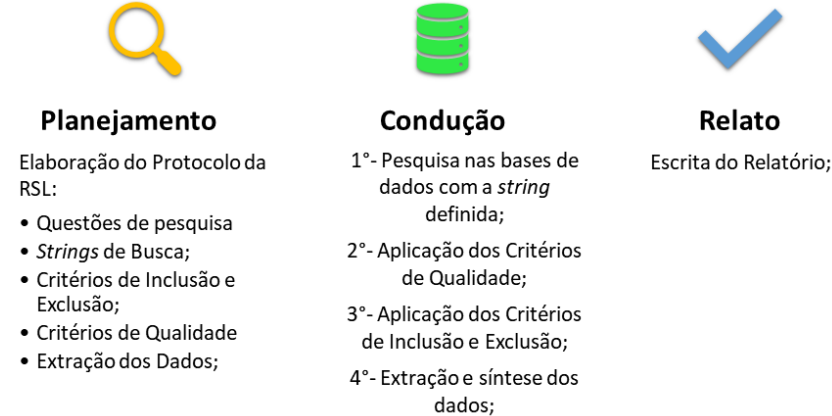

Fonte: Autores (2021)

$\mathrm{Na}$ etapa de planejamento foram definidas as questões de pesquisa, a string de busca automática nas bases de dados, os critérios de inclusão e exclusão, os critérios de qualidade e os dados a serem extraídos dos artigos. Durante a etapa de condução, na aplicação dos critérios de inclusão e exclusão, fez-se uso do software StArt, cuja finalidade de uso refere-se ao auxílio no desenvolvimento de revisões sistemáticas, oferecendo recursos que simplificam o processo.

\subsection{Questões de Pesquisa}

O objetivo desta RSL é averiguar o estado da arte a respeito da classificação dos EAs por técnicas inteligentes. Especificamente, busca-se analisar a eficiência dos algoritmos inteligentes de classificação, os dados de entrada considerados, as plataformas mais utilizadas para identificação dos estilos e, por fim, os métodos e critérios aplicados para avaliar a eficiência dos algoritmos inteligentes.

Com base nisso, as seguintes questões de pesquisa foram definidas: QP01 Quais os dados de entrada (atributos) utilizados para realizar uma classificação dos estilos de aprendizagem?; QP02 - Quais as plataformas mais adotadas para a implementação do algoritmo de classificação dos estilos de aprendizagem?; QP03 Quais métricas foram aplicadas para avaliar a eficácia das técnicas inteligentes empregadas na classificação dos estilos de aprendizagem?; QP04 - Qual é a eficiência dos algoritmos de classificação/detecção para estilos de aprendizagem?. 


\subsection{Strings de Busca}

Definidas as questões de pesquisa, foram elaboradas strings de busca usando as palavras chaves derivadas das questões de pesquisa. As strings foram aplicadas para uma busca automática nas bases de dados ACM, IEEE Xplore, Scopus, Science Direct e Web of Science (mostradas na Tabela 1). Especificamente, a string usada na base Science Direct sofreu uma variação por conta do suporte à quantidade de termos e operadores booleanos da base.

Tabela 1 - Strings usadas nas buscas nas bases de dados

\begin{tabular}{c|l}
\hline Base de Dados & \multicolumn{1}{|c}{ String de Busca } \\
\hline \multirow{3}{*}{ ACM } & [[All: "learning style"] OR [All: "learning method"] OR [All: "learning \\
IEE Xplore & [[All: "computational intelligence"] OR [All: "smart techniques"] OR [All: \\
Scopus & "artificial intelligence"] OR [All: "machine learning"]] AND [All: "student"] \\
Web of Science & AND [[All: "classification of learning"] OR [All: "detection of learning"] OR \\
& [All: "prediction of learning"] OR [All: "identification of learning"]] \\
\hline \multirow{5}{*}{$\begin{array}{l}\text { Science Direct } \\
\end{array}$} & $\begin{array}{l}\text { ("learning style" OR "learning techniques") AND ("Computational intelligence" } \\
\text { "classification of learning" OR "detection of learning" OR "identification of }\end{array}$ \\
& learning")
\end{tabular}

Fonte: Autores (2021)

\subsection{Critérios de Inclusão e Exclusão}

Após a busca nas bases de dados, seguiu-se para a etapa de aplicação dos critérios de inclusão e exclusão dos trabalhos: I01 - Artigos que apresentam técnicas inteligentes para detecção dos estilos de aprendizagem; I02 - Artigos em Inglês, Português e Espanhol; I03 - Artigos publicados em conferências, revistas e jornais. Já os critérios de exclusão são: E01 - Artigo que não possua relação a técnicas de EAs; E02 Artigos não desenvolvidos entre 2015 e 2020; E03 - Artigos incompletos; E04 Trabalhos duplicados; E05 - Trabalhos não disponíveis na web; E06 - Artigos secundários.

A aplicação dos critérios de inclusão e exclusão se deu em duas etapas: na primeira etapa foi realizada a leitura, por ambos os autores, do título, resumo e palavraschave dos trabalhos retornados pelas pesquisas nas bases de dados. Após a leitura, verificou-se a validade de cada trabalho diante dos critérios de inclusão e exclusão. Caso algum trabalho se enquadrasse em pelo menos um critério de exclusão ou não se enquadrasse em todos os critérios de inclusão o trabalho era eliminado da RSL. 
$\mathrm{Na}$ segunda etapa, foi realizada a leitura completa dos trabalhos selecionados e feita uma reavaliação para averiguar se atendem aos critérios já estabelecidos na primeira etapa.

\subsection{Qualificação dos trabalhos e extração dos dados}

Com os trabalhos já selecionados, foi iniciada a qualificação dos trabalhos e a extração dos dados. Essas duas etapas foram feitas de forma paralela devido à grande quantidade de trabalhos, a fim de otimizar o tempo. Então, à medida que os dados foram extraídos, os artigos também foram sendo qualificados. Os critérios de qualidade são os seguintes: QL01: Os objetivos do trabalho são apresentados de forma clara e concisa?; QL02: As metodologias adotadas são apresentadas de forma clara e concisa?; QL03: Foram realizadas discussões claras a respeito dos resultados obtidos?; QL04: A teoria dos EAs utilizada está detalhada? QL05: Todos os termos técnicos que são apresentados ao longo do trabalho são discutidos no capítulo de referencial teórico?; QL06: Está clara a eficiência do(s) algoritmo(s) de classificação/detecção de EAs?; QL07: Está claro qual(is) o(s) dado(s) de entrada (atributos) usados para realizar a classificação dos estilos de aprendizagem?; QL08: É(são) apresentada(s) a(s) plataforma(s) adotada(s) para implementação do algoritmo de classificação dos EAs?; QL09: É evidenciado quais os métodos/critérios foram aplicados para avaliar a eficácia dos EAs classificados pelos sistemas inteligentes?; QL10: A proposta/ferramenta foi validada em um cenário real?; QL11: A técnica de IA abordada está explicitamente descrita?.

O processo de avaliação dos critérios de qualidade dos trabalhos selecionados procedeu mediante a leitura completa dos trabalhos e mensuração de uma pontuação. Os artigos obtiveram nota 0 quando não responderam ao critério de qualidade; 0,5 quando responderam parcialmente; e 1 quando responderam totalmente. A nota total máxima que cada artigo poderia obter era 11, devido a quantidade de critérios de qualidade.

$\mathrm{Na}$ extração dos dados utilizou-se um formulário com as informações a serem coletadas dos artigos, as quais são: EXT01: ID; EXT02: Título; EXT03: Detalhes da publicação; EXT04: Breve resumo do artigo; EXT05: Dados de entrada (atributos) usados para classificação dos EAs; EXT06: Plataformas (WEB, mobile, desktop) de implementação da ferramenta de predição dos estilos de aprendizagem; EXT07: Teoria de estilos de aprendizagem utilizada; EXT08: Técnica inteligente usada para classificar os estilos de aprendizagem; EXT09: Métricas aplicadas para avaliação do desempenho 
do algoritmo inteligente; EXT10: Desempenho do algoritmo de classificação/detecção para estilos de aprendizagem; EXT11: País onde foi realizada a pesquisa. Os dados referentes a EXT01, EXT02, EXT03 e EXT04 são dados usados para organização dos artigos na tabela de extração. Sendo o EXT01 um número fornecido pelo StArt para identificação do trabalho e o EXT04 um curto resumo feito pelos autores a fim de explanar de forma rápida e objetiva sobre as contribuições de cada pesquisa.

Posterior à fase de qualificação e extração, seguiu-se para a síntese e análise dos dados. Esta etapa está descrita na próxima seção, onde foram especificados os dados relacionados às questões de pesquisa e outros dados que também foram coletados de forma a complementar aqueles das questões de pesquisa.

\section{RESULTADOS E DISCUSSÕES}

Nesta seção são apresentados os resultados finais relacionados à RSL, ela é dividida em 3 subseções: na 3.1 são apresentados os resultados gerais das buscas e dos critérios de qualidade, na 3.2 são apresentadas as respostas das questões de pesquisas, e por fim, na 3.3 são expostos outros resultados adicionais que agregam ao tema da pesquisa em questão.

\subsection{Resultados da Busca e Critérios de Qualidade}

Nesta subseção, são discutidos os resultados gerais, obtidos no processo de seleção de trabalhos advindos das bases de dados, bem como o resultado da avaliação dos critérios de qualidade dos trabalhos selecionados que atenderam aos critérios de inclusão.

\subsubsection{Resultados da Busca e Seleção dos Trabalhos nas Bases de Dados}

O processo de pesquisa nas cinco bases de dados resultou em 565 trabalhos préselecionados. Desses, 506 não se enquadram em algum dos critérios de inclusão ou se enquadram em pelo menos um critério de exclusão como, por exemplo, a ocorrência de duplicidade de 20 artigos. A lista com os trabalhos selecionados pode ser verificada no link $^{2}$. a A Tabela 2 apresenta a quantidade de trabalhos retornados nas bases de dados e a quantidade restante após aplicação dos critérios de inclusão e exclusão.

\footnotetext{
2 https://sites.google.com/alunos.ufersa.edu.br/rsl-estilos-de-aprendizagem/home
} 
Tabela 2 - Resultado do processo de seleção dos trabalhos nas bases de dados

\begin{tabular}{|c|c|c|}
\hline Base de Dados & Artigos Pré-Selecionados & Artigos Selecionados \\
\hline ACM & 21 & 1 \\
\hline IEEE & 4 & 1 \\
\hline Scopus & 380 & 54 \\
\hline ScienceDirect & 157 & 2 \\
\hline Web of Science & 3 & 1 \\
\hline Total & $\mathbf{5 6 5}$ & $\mathbf{5 9}$ \\
\hline
\end{tabular}

Fonte: Autores (2021)

\subsubsection{Resultados dos Critérios de Qualidade}

Nesta subseção são descritos os resultados das pontuações dos trabalhos de acordo com os critérios de qualidade. Essa explanação se dá também por gráficos e tabelas através de várias perspectivas. Primeiro, a pontuação de cada trabalho, que também está disponível no site ${ }^{3}$ deste trabalho, depois, os artigos são divididos em quatro categorias qualitativas (Figura 2), e, por fim, a soma total das notas de cada critério de qualidade. A pontuação máxima que cada trabalho pode obter é 11.

A partir dessas pontuações, os autores construíram uma classificação qualificatória para os artigos. Nessa classificação, os que apresentam pontuação abaixo de 5,5, ou seja, metade da nota total que os artigos poderiam obter, têm qualidade insatisfatória, entre 5,5 e 7,5, pouco satisfatória, entre 8,0 e 9,0, satisfatória, e entre 9,5 e 11, muito satisfatória. Não houve trabalhos com qualidade insatisfatória.

Figura 2 - Quantidade de trabalhos para cada classificação de acordo com a pontuação da qualidade obtida

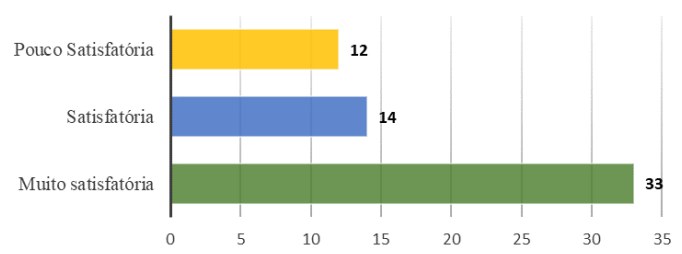

Fonte: Autores (2021)

Também foram avaliadas as notas totais de cada critério de qualidade, ou seja, a nota total que cada critério de qualidade obteve, somando-se as notas do respectivo critério em cada artigo. A nota máxima possível era de 59, no caso em que todos os 59 artigos obtivessem nota 1 em um determinado critério. Essas notas estão explicitadas na Tabela 3.

\footnotetext{
3 https://sites.google.com/alunos.ufersa.edu.br/rsl-estilos-de-aprendizagem/home
} 
Tabela 3 - Notas totais calculadas para cada critério de qualidade

\begin{tabular}{|c|c|c|c|c|c|c|c|}
\hline Critério & Nota & Critério & Nota & Critério & Nota & Critério & Nota \\
\hline QL01 & 56,5 & QL04 & 52 & QL07 & 48 & QL10 & 54 \\
\hline QL02 & 51,5 & QL05 & 50 & QL08 & 45,5 & QL11 & 58 \\
\hline QL03 & 43 & QL06 & 46,5 & QL09 & 45,5 & & \\
\hline
\end{tabular}

Fonte: Autores (2021)

Como pode ser observado na Tabela 3, o critério de qualidade com maior pontuação foi o QL11, relacionado à técnica de IA utilizada, que obteve 58 pontos. Em segundo, o QL01, com 56,5, o que indica que a maioria dos trabalhos apresentou os seus objetivos de forma clara e concisa. Os demais critérios obtiveram nota entre 43 e 54 pontos, sendo o QL03, que trata da discussão dos resultados obtidos, o que obteve menor pontuação.

\subsection{Respostas às Questões de Pesquisa}

Nesta subseção são apresentados os resultados relacionados às quatro questões de pesquisa que esta RSL busca responder.

3.2.1 Dados de entrada (atributos) utilizados para realizar a classificação dos estilos de aprendizagem (QP01)

Esta questão se refere aos dados considerados para classificar e/ou detectar os EAs, isto é, os atributos que servem de entrada para o algoritmo de classificação ou detecção. Os atributos primários, obtidos na etapa de extração, apresentaram grande variedade de informações e multiplicidade de termos, por isso foram classificados em categorias mais gerais, descritas a seguir, para facilitar a visualização dos resultados.

\subsubsection{Interações do aluno com objetos de aprendizagem (AT01)}

Corresponde aos diversos tipos de objetos de aprendizagem acessados pelo aluno na plataforma. Estão incluídos nessa categoria o tempo gasto e o número de acessos ao objeto de aprendizagem (OA), além das diversas maneiras de interação dos alunos com eles. Os OAs são os diversos tipos de material que os alunos têm acesso no ambiente de aprendizagem, como vídeos, PDFs, áudios, entre muitos outros.

\subsubsection{Desempenho e nível de participação do aluno no curso (AT02)}

Os atributos relacionados ao desempenho do aluno em atividades dentro do curso, como a pontuação em testes e avaliações, desempenho acadêmico anterior, conhecimento prévio sobre o assunto do curso, assiduidade e atenção do aluno no curso.

\subsubsection{Questionário (AT03)}

Se refere aos questionários elaborados pelos autores dos artigos que foram usados para coletar os dados de entrada para os algoritmos, bem como os tradicionais 
questionários referentes às diversas teorias dos EAs, como VARK (Visual, Aural, Read, Kinesthetic), Cuestionario Honey-Alonso de Estilos de Aprendizaje (CHAEA) e o Felder-Silverman Learning Style Model (FSLSM).

\subsubsection{Comportamentos Físicos e Fisiológicos (AT04)}

Correspondem a fatores fisiológicos e reações físicas que os alunos tiveram durante o processo de ensino-aprendizagem. São eles: os batimentos cardíacos, pressão arterial, atividade cerebral, movimento dos olhos e expressões faciais em reação, por exemplo, aos objetos e/ou métodos de ensino que estavam sendo aplicados.

\subsubsection{Ferramentas de contato com outros usuários (AT05)}

São os meios adotados pelos alunos para se comunicar com colegas, professores ou outras pessoas quando têm alguma dúvida, podem ser chats, quadros de discussão, email e outros.

\subsubsection{Pessoa de contato em dificuldade (AT06)}

Trata-se da pessoa com quem, durante a realização de uma tarefa, o aluno entra em contato se tiver alguma dificuldade. Podem ser colegas de curso, professores ou outras pessoas.

\subsubsection{Desempenho em Jogos (AT07)}

Refere-se a pontuação dos alunos em jogos matemáticos para classificá-los de acordo com seu EA. Os jogos consistiam em responder corretamente ou não às questões de operações matemáticas básicas, como adição e subtração, e apresentavam dois níveis de dificuldade.

\subsubsection{Interações com software e/ou hardware (AT08)}

Neste caso foram consideradas interações bem específicas dos usuários com o sistema de aprendizagem que não se enquadram em outras categorias, esses atributos correspondem aos utilizados por Crockett et al., (2017). Essa categoria também se refere ao monitoramento dos padrões de comportamento do mouse e teclado, e o tipo e tempo de uso das redes sociais.

\subsubsection{Sequências de aprendizagem (AT09)}

Conjunto de diferentes OAs usados durante uma sessão do curso, as sequências também contém outras informações relativas, como o id ${ }^{4}$ do aluno, o id da sessão, id da página acessada e o tempo gasto em cada página e OA.

\footnotetext{
${ }^{4}$ Id é um código de identificação.
} 


\subsubsection{Dados demográficos (AT10)}

Características pessoais dos alunos, como idade, sexo, estado civíl e local onde vivem.

A Figura 3 mostra o número de ocorrências para cada categoria de atributos citada. A categoria de atributos com mais ocorrências foi AT01, com 63 ocorrências. O AT02 fica em segundo lugar com 16, seguido dos AT03, com 7, e AT09, 6. Os AT04 e AT08 foram considerados 3 vezes. Os AT01, AT05, AT07 foram utilizados duas vezes cada, e o AT06 uma. Houveram 6 artigos que não especificaram os atributos usados.

Figura 3 - Número de ocorrências de cada categoria de atributos

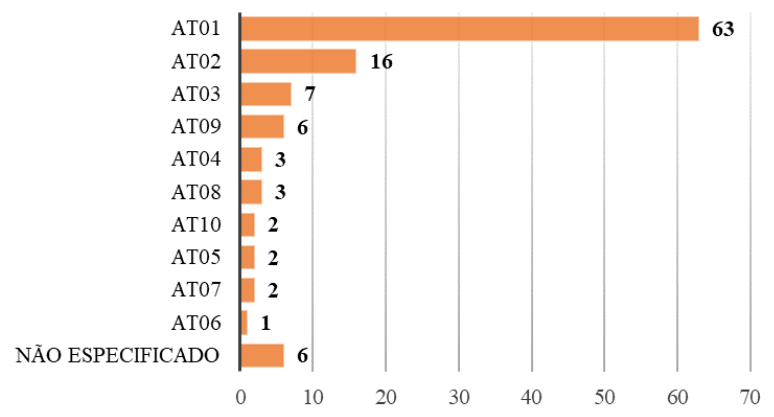

Fonte: Autores (2021)

3.2.2 Plataformas mais adotadas para a implementação do algoritmo de classificação do estilos de aprendizagem (QP02)

As plataformas usadas podem ser do tipo $W E B$, mobile ou desktop. A Figura 4 mostra os resultados encontrados. Como pode ser observado na Figura 4, a plataforma mais explorada para implementação dos algoritmos foi a $W E B$ com 47 artigos, seguida da desktop com 4, e da mobile com 1.

Exemplos de plataformas WEB usadas nos artigos são: LMS (Learning Management Systems), ITS (Intelligent Tutoring System), MOODLE (Modular ObjectOriented Dynamic Learning Environment) e MOOCS (Massive Open Learning Courses). Essas plataformas são exemplos de ambientes virtuais de aprendizagem, usados como suporte para os diversos tipos de cursos online.

Esses ambientes serviam de suporte para a coleta de arquivos de $\log$ que continham as informações de uso dos alunos no ambiente. Essas informações são os atributos, mencionados na seção anterior, que servem, então, para predizer o EA do aluno de acordo com a abordagem proposta. Após a predição dos EAs, algumas plataformas realizavam adaptações em seus escopos, com o propósito de fornecer material de estudo de acordo com o EA do aluno. 
Figura 4 - Número de utilizações das plataformas de implementação da ferramenta de predição dos estilos de aprendizagem.

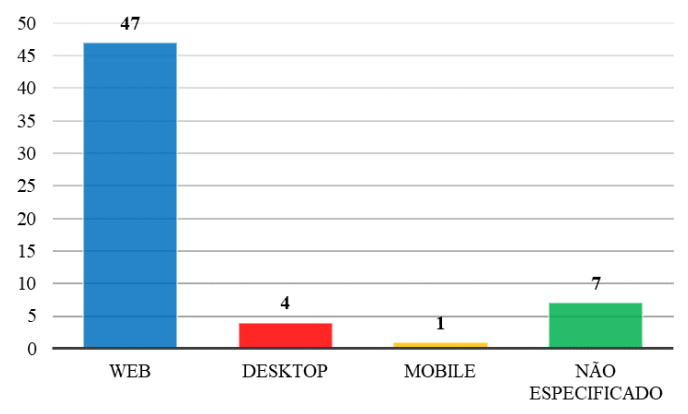

Fonte: Autores (2021)

\subsubsection{Quais métricas foram aplicadas para avaliar a eficácia das técnicas} inteligentes empregadas na classificação dos estilos de aprendizagem (QP03)?

Esta subseção apresenta os resultados relacionados à (QP3), tendo como objetivo averiguar quais as métricas mais exploradas na literatura para avaliar a eficácia das técnicas de IA usadas para classificação dos EAs. A Figura 5 apresenta os resultados relacionados a $\mathrm{QP} 03$.

Figura 5 - Métricas aplicadas para avaliar o desempenho das técnicas inteligentes.

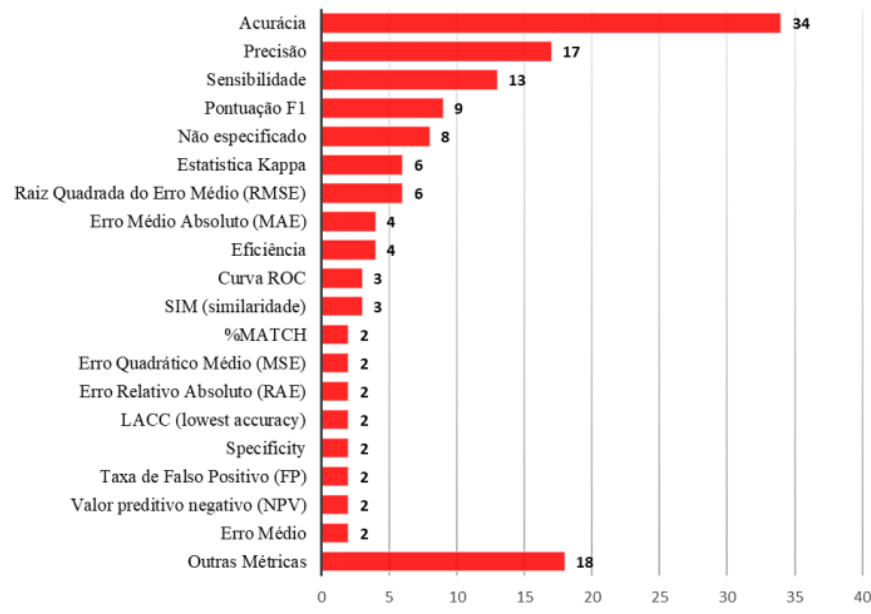

Fonte: Autores (2021)

As métricas mais adotadas foram Acurácia, 34 vezes, Precisão 17, Sensibilidade 13, e Pontuação F1 (Fl-score), 9. Além disso, 8 trabalhos não especificaram as métricas utilizadas para avaliar a precisão da classificação gerada pela técnica inteligente. A categoria outras métricas é o conjunto de todas as métricas que foram utilizadas apenas uma vez.

Vale ressaltar que diversos trabalhos usaram mais de uma métrica para avaliar o desempenho das técnicas propostas, agregando assim, maior confiabilidade e 
credibilidade à técnica. Os dados expostos apresentam-se de maneira relevante para nortear pesquisadores ou desenvolvedores a respeito das métricas mais utilizadas e que podem ser adotadas para avaliar a eficiência de técnicas inteligentes.

3.2.4 Qual é a eficiência dos algoritmos de classificação/detecção para estilos de aprendizagem (QP04)?

O objetivo da QP04 tratada nesta subseção é apresentar o quão eficiente são as técnicas inteligentes citadas nos trabalhos revisados na classificação dos EAs, ou seja, é evidenciado os resultados das métricas tratadas na subseção 3.2.3 juntamente com sua respectiva técnica. Os resultados obtidos podem ser visualizados no presente site $^{5}$, onde é apresentado a média percentual da performance de cada técnica inteligente e com sua respectiva métrica. Para obtenção destes valores foi realizada a média percentual dos desempenhos das técnicas inteligentes relacionadas a cada métrica utilizada.

As técnicas inteligentes utilizadas nos trabalhos revisados estão descritas na seção 3.3.1, dentre elas pode-se destacar: Case-Based Reasoning (CBR), que obteve uma média percentual de $99.50 \%$ de acurácia, sendo esta a métrica mais utilizada nos trabalhos revisados; C4.5, que atingiu uma média percentual de $98.60 \%$ de precisão, sendo ela a segunda métrica mais adotada; e Fuzzy C-means (FCM), que alcançou a média percentual de $99.44 \%$ de sensibilidade.

\subsection{Outros Resultados}

Nesta seção são apresentados resultados adicionais obtidos a partir da RSL que não foram selecionados como questões centrais desta revisão, mas que contribuem para o entendimento das questões de pesquisas trabalhadas e para a melhor análise do estado da arte relacionadas às técnicas inteligentes para classificação dos EAs.

\subsubsection{Técnicas inteligentes}

Nesta subseção são apresentados os resultados relacionados às técnicas inteligentes mais adotadas para classificar os EAs. Tais resultados são explicitados na Figura 6. A partir da Figura 6 é possível observar a variedade de técnicas inteligentes presentes na literatura adotadas para classificar os EAs, totalizando 50 técnicas distintas. A técnica inteligente que se sobressaiu entre as demais foi a Artificial Neural Network (ANN) com 11 utilizações, seguida da técnica Naive Bayes, com 10, e posteriormente

\footnotetext{
5 https://sites.google.com/alunos.ufersa.edu.br/rsl-estilos-de-aprendizagem/home
} 
pela técnica $K$-means $(\mathrm{KM})$, com 9. A categoria outras métricas, que obteve 32 usos, é composta por técnicas que apresentaram somente uma aplicação.

Figura 6 - Técnicas inteligentes utilizadas para classificar os EAs.

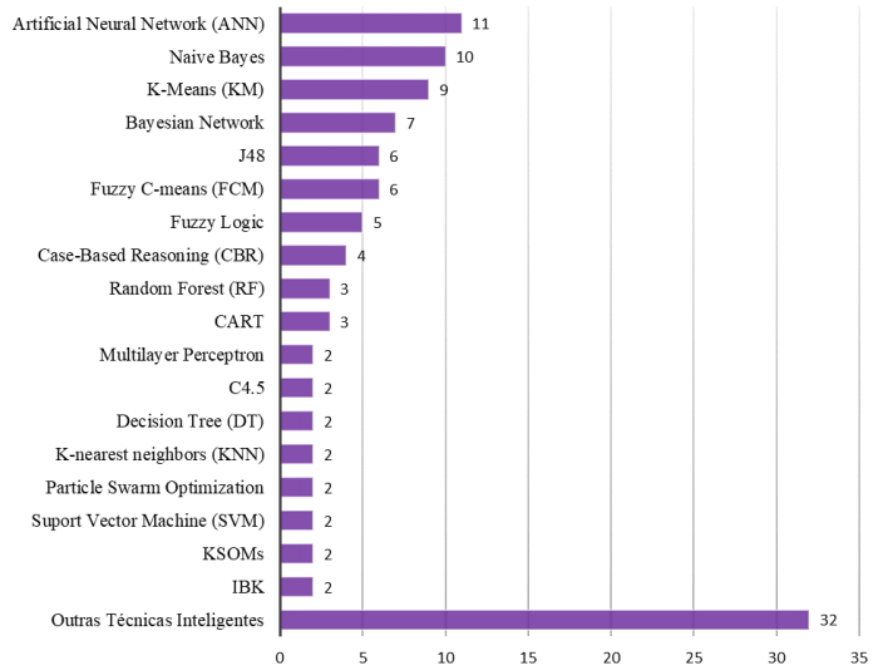

Fonte: Autores 2021

Vale ressaltar que diversos trabalhos utilizaram a junção de técnicas distintas para classificar os EAs, com o intuito de otimizar os resultados obtidos, e em alguns trabalhos foram realizadas comparações entre as técnicas propostas com outras técnicas, para averiguar qual a que apresentava o melhor desempenho e comprovar a eficiência da técnica desenvolvida.

\subsubsection{Teoria de estilos de aprendizagem}

As teorias de EAs são importantes pois elas definem os tipos de estilos existentes com base em diferentes fatores dependentes da teoria. Cada uma divide os EAs em diferentes números e tipos de categorias. A Figura 7 mostra as teorias de EAs mais exploradas nas abordagens propostas pelos artigos revisados.

A teoria de EAs mais empregada foi o FSLSM, abordado em 38 trabalhos. Em seguida, temos o modelo VARK com 8 usos, o modelo de Kolb com 4, os modelos Honey-Alonso e Honey-Mumford com 3, e, por último o Myers-Briggs type Indicator (MBTI) ou Tipologia de Myers Briggs, o CHAEA-32 e o CHAEA-JQ que foram abordados apenas uma vez. Um dos artigos não especificou o modelo preferido.

Os últimos dois questionários CHAEA (CHAEA-32 e CHAEA-JQ), foram mencionados de forma separada pois, como o próprio nome indica, eles apresentam adaptações em relação ao CHAEA original. O CHAEA-32 tem um número reduzido de questões e o CHAEA-JQ é adaptado para crianças. O BAJAJ e SHARMA (2018) se basearam em duas teorias na sua abordagem, o FSLSM e o modelo de Kolb. 
Figura 7 - Teorias de EAs utilizadas nas abordagens.

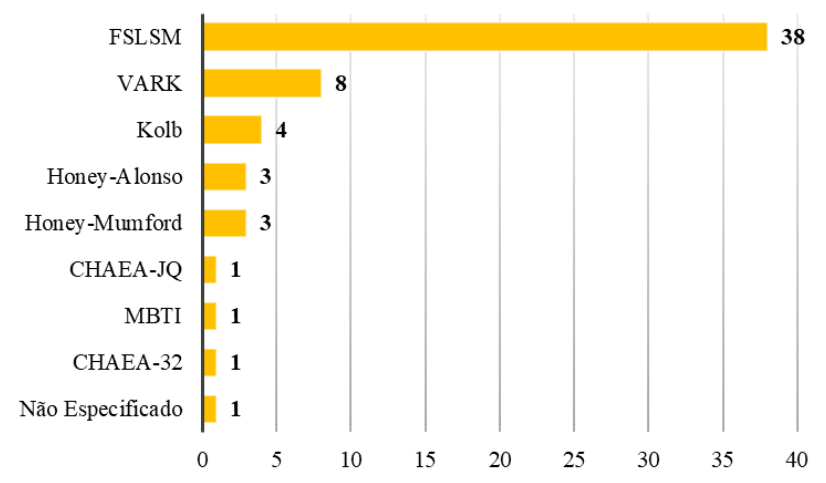

Fonte: Autores (2021)

\subsubsection{Países onde os dados foram coletados}

Também foram investigados em quais países as pesquisas ocorreram. O país citado em mais trabalhos foi o Marrocos, em 7 trabalhos, seguido da Indonésia e Peru, com 5 trabalhos cada, Grécia, 4, e Brasil, 3. Depois, temos Argélia, Equador, Estados Unidos, Índia, Malásia e Paquistão, citados em 2 trabalhos. Por fim, Áustria, China, Colômbia, Egito, Inglaterra, Arábia Saudita, Nigéria, Portugal, Reino Unido e Tailândia foram citados apenas uma vez. 13 trabalhos não especificaram o país.

\section{CONCLUSÃO}

A identificação dos EAs, como enfatizada por Schimitt and Domingues (2016), permite o desenvolvimento de estratégias pedagógicas eficazes alinhadas às necessidades do aluno. O objetivo deste trabalho foi realizar um levantamento das pesquisas existentes com relação às técnicas inteligentes para classificação dos EAs. Para cumprir o referido objetivo foi conduzida uma RSL norteada pelo protocolo proposto por Kitchenham and Charters (2007).

De acordo com os resultados, os dados de entrada (atributos) utilizados para realizar a classificação dos EAs são as interações do aluno com objetos de aprendizagem, com 63 estudos, respondendo a QP01. Tais objetos de aprendizado, em sua maioria, são disponibilizados nas plataformas de ensino acessadas pelo aluno. Dentre essas plataformas, tratadas na QP02, as mais usadas para a implementação do algoritmo de identificação dos EAs foram as plataformas $W E B$, aparecendo em 47 estudos, por ser o meio mais fácil de se acessar os ambientes de estudo pelos diferentes 
tipos de alunos (ensino fundamental, médio, superior) e por sua escalabilidade para implementação das ferramentas de classificação.

A acurácia foi a métrica mais utilizada para avaliar a eficácia dos EAs propostos por técnicas inteligente QP03, sendo aplicada em 37 estudos, sua adoção dentre a maioria dos trabalhos se deu precedida de outras métricas, garantindo assim melhor qualidade na avaliação dos EAs identificados pelas técnicas inteligentes. E por fim, a técnica inteligente CBR apresentou maior eficiência na classificação dos EAs apresentando uma acurácia de 99.50, respondendo assim a QP04.

Baseando-se nos resultados desta RSL, almeja-se como trabalho futuro o desenvolvimento de uma nova abordagem para classificar EAs, explorando as áreas menos abordadas e os pontos fortes de cada proposta dos artigos investigados.

\section{REFERÊNCIAS}

BAJAJ, R.; SHARMA, V. Smart Education with artificial intelligence based determination of learning styles. Procedia computer science, [s. l.], v. 132, p. 834-842, 2018.

CROCKETT, K.; LATHAM, A.; WHITTON, N. On predicting learning styles in conversational intelligent tutoring systems using fuzzy decision trees. International Journal of Human-Computer Studies, [s. l.], v. 97, p. 98-115, 2017.

DUTSINMA, L. I. F.; TEMDEE, P. VARK learning style classification using decision tree with physiological signals. Wireless Personal Communications, [s. l.], p. 1-22, 2020 .

EL AISSAOUI, O.; EL MADANI, Y. E. A.; OUGHDIR, L.; EL ALLIOUI, Y. A fuzzy classification approach for learning style prediction based on web mining technique in e-learning environments. Education and Information Technologies, [s. l.], v. 24, n. 3, p. 1943-1959, 2019.

KITCHENHAM, B.; CHARTERS, S. Guidelines for performing systematic literature reviews in software engineering. [s. l.], 2007.

SCHMITT, C. da S.; DOMINGUES, M. J. C. de S. Estilos de aprendizagem: um estudo comparativo. Avaliação: Revista da Avaliação da Educação Superior (Campinas), [s. l.], v. 21, p. 361-386, 2016. 